\title{
THE SPECTROSCOPIC ORBIT OF $\phi$ CYGNI, A SYSTEM WITH TWO LATE-TYPE GIANTS
}

\author{
Francis C. Fekel ${ }^{1}$ \\ RESUMEN
}

Se han determinado elementos orbitales mejorados para $\phi$ Cygni, mediante 47 nuevas observaciones espectroscópicas. El período es 434.14 días y la excentricidad 0.559 . Con las masas mínimas y la inclinación de la órbita visual obtenemos masas de 2.16 y $2.06 \mathrm{M}_{\odot}$ para las dos gigantes. La pequeña diferencia de magnitudes $(0.25 \mathrm{mag})$ y el cociente de masas (1.044) indican que ambas gigantes se encuentran en la fase evolutiva del quemado del helio.

\begin{abstract}
Improved orbital elements for $\phi$ Cygni have been determined from 47 new high-dispersion spectroscopic observations. The period and eccentricity are 434.14 days and 0.559 , respectively. Minimum masses plus the inclination of the visual orbit result in masses of 2.16 and $2.06 \mathrm{M}_{\odot}$ for the two giants. The small magnitude difference of $0.25 \mathrm{mag}$ and a mass ratio of 1.044 indicate that both giants are most likely in the helium-burning phase of their evolution.
\end{abstract}

Key Words: BINARIES: CLOSE - BINARIES: SPECTROSCOPIC

\section{INTRODUCTION}

The star $\phi$ Cygni is a double-lined binary that consists of a pair of $\mathrm{K} 0$ giants. Such a spectroscopic pair of giants is quite rare. The separation of the components and hence the orbital period must be great enough so that mass transfer does not substantially alter the evolution of the system. The components also must have similar masses, so that the stars arrive on the giant branch at about the same time. While the initial spectrum of $\phi \mathrm{Cyg}$, obtained in 1907 (Campbell 1909), showed double lines, over 50 years elapsed before Rach \& Herbig (1961) computed the first orbit for the system. They determined a period of 434.09 days and minimum masses of 2.36 and $2.27 \mathrm{M}_{\odot}$. Observations with the Mark III Optical Interferometer enabled Armstrong et al. (1992) to determine visual orbital elements for the system. Considering both the interferometric and spectroscopic observations, they adopted a set of best-fit orbital elements that included an orbital inclination of $78^{\circ} .4$. ¿From the combined results they determined masses of 2.54 and $2.44 \mathrm{M}_{\odot}$ for the components and a distance to the system of $80.8 \mathrm{pc}$. Discrepancies between the spectroscopic and visual elements suggested that additional spectroscopic observations would be useful.

\footnotetext{
${ }^{1}$ Center of Excellence in Information Systems, Tennessee State University, Nashville, TN, USA. email: fekel@evans.tsuniv.edu
}

\section{OBSERVATIONS AND REDUCTIONS}

From 1991 June to 2002 September, 44 highresolution spectroscopic observations were acquired with the Kitt Peak National Observatory (KPNO) coudé feed telescope, coudé spectrograph, and a TI $\mathrm{CCD}$ detector. Forty-two of the spectrograms were centered in the red at $6430 \AA$ and two were at $6700 \AA$. Those observations all have a resolution of $0.21 \AA$, cover a wavelength range of about $80 \AA$, and have typical signal-to-noise ratios of 200 . Three additional observations were obtained at other wavelengths by other observers, resulting in 47 spectra that showed double lines.

Radial velocities were determined with the IRAF cross-correlation program FXCOR (Fitzpatrick 1993). The star $\beta$ Aquilae was used as the reference star for the velocities of $\phi$ Cyg. From my unpublished results, it has a radial velocity of -40.2 $\mathrm{km} \mathrm{s}^{-1}$. In many instances the lines of the two stars were not completely resolved, and so a double Gaussian fit was made to the resultant cross-correlation peak of the blended lines.

\section{DISCUSSION}

The current observations span more than 9 orbital cycles and include the recent 2002 September periastron passage. Figure 1 compares the new radial velocities with the computed orbit, determined from the orbital elements listed in Table 1. Armstrong et al. (1992) noted difficulties in reconciling 


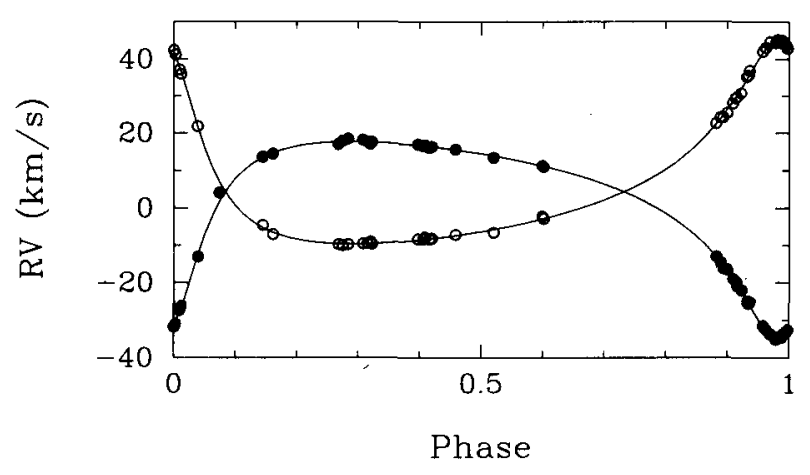

Fig. 1. A comparison of the radial velocities and computed orbit for $\phi$ Cyg. Solid circles represent the primary, open circles, the secondary.

TABLE 1

ORBITAL ELEMENTS OF $\phi$ CYG

\begin{tabular}{|c|c|}
\hline Parameter & Value \\
\hline$P$ (days) & $434.14 \pm 0.04$ \\
\hline$T$ (HJD) & $2,450370.8 \pm 0.2$ \\
\hline$\gamma\left(\mathrm{km} \mathrm{s}^{-1}\right)$ & $4.35 \pm 0.06$ \\
\hline$K_{A a}\left(\mathrm{~km} \mathrm{~s}^{-1}\right)$ & $26.24 \pm 0.08$ \\
\hline$K_{A b}\left(\mathrm{~km} \mathrm{~s}^{-1}\right)$ & $27.39 \pm 0.07$ \\
\hline$e$ & $0.559 \pm 0.002$ \\
\hline$\omega_{A a}(\operatorname{deg})$ & $208.5 \pm 0.5$ \\
\hline$a_{A a} \sin i(\mathrm{~km})$ & $129.9 \pm 0.4 \times 10^{6}$ \\
\hline$a_{A b} \sin i(\mathrm{~km})$ & $135.6 \pm 0.4 \times 10^{6}$ \\
\hline$m_{A a} \sin ^{3} i\left(\mathrm{M}_{\odot}\right)$ & $2.03 \pm 0.01$ \\
\hline$m_{A b} \sin ^{3} i\left(\mathrm{M}_{\odot}\right)$ & $1.94 \pm 0.01$ \\
\hline
\end{tabular}

a couple of their visual orbital elements, specifically the eccentricity and to a lesser extent the longitude of periastron, with those of the spectroscopic orbit of Rach \& Herbig (1961). The new spectroscopic elements that are in common with those of Armstrong et al. (1992) agree within their uncertainties and remove such discrepancies. The new elements confirm the mass ratio of the two giants that was determined by Rach \& Herbig (1961), but the minimum masses computed from the current spectroscopic solution are $\sim 0.3 \mathrm{M}_{\odot}$ smaller. Basic data for $\phi$ Cyg are listed in Table 2 .
TABLE 2

FUNDAMENTAL PARAMETERS

\begin{tabular}{lcl}
\hline Parameter & Value & Reference \\
\hline$V(\mathrm{mag})$ & 4.68 & ESA (1997) \\
$B-V(\mathrm{mag})$ & 0.971 & ESA (1997) \\
$\pi\left(^{\prime \prime}\right)$ & $0.01300 \pm 0.00059$ & ESA (1997) \\
$\pi_{\text {orb }}\left(^{\prime \prime}\right)$ & $0.01308 \pm 0.00022$ & This work \\
$a(\mathrm{AU})$ & $1.812 \pm 0.005$ & This work \\
$\mathrm{M}_{A}\left(\mathrm{M}_{\odot}\right)$ & $2.16 \pm 0.01$ & This work \\
$\mathrm{M}_{B}\left(\mathrm{M}_{\odot}\right)$ & $2.06 \pm 0.01$ & This work \\
$\mathrm{M}_{A} / \mathrm{M}_{B}$ & $1.044 \pm 0.004$ & This work \\
$\mathrm{L}_{A}\left(\mathrm{~L}_{\odot}\right)$ & $48.3 \pm 3.1$ & This work \\
$\mathrm{L}_{B}\left(\mathrm{~L}_{\odot}\right)$ & $38.4 \pm 2.5$ & This work \\
\hline
\end{tabular}

At $6430 \AA$ the line strengths of the two components are slightly different and result in a magnitude difference of about 0.25. According to Schaller et al. (1992), a solar-abundance star of $2 \mathrm{M}_{\odot}$ crosses the Hertzsprung Gap and ascends the first giant branch to helium ignition in about 300 million years. The magnitude difference of 0.25 and a mass dititrence of $0.1 \mathrm{M}_{\odot}$ between the components suggest that both stars are beginning their helium-burning phase of evolution, as argued by Armstrong et al. (1992).

This research at Tennessee State University is supported in part by NASA grant NCC 5-511 and NSF grant HRD-9706268.

\section{REFERENCES}

Armstrong, J. T., et al. 1992, AJ, 104, 2217

Campbell, W. W. 1909, ApJ, 29, 224

ESA. 1997, The Hipparcos and Tycho Catalogues (ESA SP-1200), (Noordwijk: ESA)

Fitzpatrick, M. J. 1993, in ASP Conf. Ser. 52, Astronomical Data Analysis Software and Systems II, ed. R. J. Hanisch, R. V. J. Brissenden, \& J. Barnes (San Francisco: ASP), 472

Rach, R. A., \& Herbig, G. H. 1961, ApJ, 133, 143

Schaller, G., Schaerer, D., Meynet, G., \& Maeder, A. 1992, A\&AS, 96, 269 